angiographic anatomy, approaches for stenting, equipment. There is also a section on management of acute stroke in this part.

The last part describes complications related to stenting, and post-procedural monitoring and follow-up.

The individual sections are comprehensive, and the text and schematic figures are of good quality. However, many of the angiographic images are dark, or poor quality, suggesting that they are either low matrix images, cine mode or fluoroscopic loop stored images, rather than high-detail multi-frame digital subtraction (DSA) images. In a field where high spatial resolution, as well as high quality angiography, are essential, as described in the equipment needed in Part II, this detracts from the otherwise good quality of the book.

At the end of Part III, there is a short section on training and credentialing, which comes after the section on reimbursement. It does not relate to the Canadian health care system, or other jurisdictions, merely the U.S. system. In my view, this is a minor deficiency. There is a larger section on training and credentialing - Chapter 8 - in Part III, which is more comprehensive, detailing training requirements for operators.

The sections on anatomy (apart from images, described above) are good, and the technique sections are also good.

The index is adequate.

Overall, this is a reasonable introduction to carotid artery stenting, allowing for the suboptimal image quality.

Donald Lee London, Ontario, Canada

Neurotrauma and Critical Care of the Brain. 2009. By Jack Jallo, Christopher M. Loftus. Published by Thieme Medical Publishers, Inc. 485 pages. C\$215 approx.

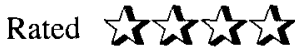

"Neurotrauma and Critical Care of the Brain" is a well compiled document on the current status of diagnosis and treatment of brain trauma. The team approach makes it possible for experts in the different subsections to write about each of those sections in an authoritative and up-to-date fashion. The subsections include:

- Epidemiology

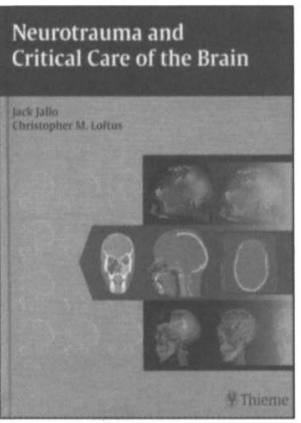

- The Signs of Brain Injury

- Management of Brain Injury

- Critical Care

- Outcome

- Socioeconomics

Subdivisions in the book are very well organized. This lends itself to the book being used as a reference for current concepts in different aspects of neurotrauma. Of particular interest is the fact that most chapters have a summary of the content. Each chapter also has extensive references.

I particularly liked the fact that the authors include experts in Critical Care. They discussed different aspects of non-invasive, and invasive monitoring. They were also able to separate concepts that are of research status from those that are of practical clinical value at this time.

"Mild brain injury" has become a major topic in recent years, especially as related to sports. This is often treated very lightly in books on brain injury. I was glad to note that a considerable space was spent on this subject, including a mention of the "Consensus Statement of the 2nd International Symposium on Concussion in Sports, Prague 2004". Readers should of course note that there is now a "Consensus Statement of the 3rd International Symposium on Concussion in Sports, Zurich 2008".

The section on "Introduction to Brain Injury Imaging" is very well done. Typical images of common types of brain injury are demonstrated. I would have liked a companion CD, or DVD, of more images that are described in the book, as well as all quoted references on the same $\mathrm{CD}$.

Overall, I highly recommend this book for all who are interested in evidence based concepts in the management of patients with traumatic brain injury.

Chris E.U. Ekong Regina, Saskatchewan, Canada

Imaging the Brain with Optical Methods. 2010. Edited by Anna W. Roe. Published by Springer. 267 pages. $C \$ 190$ approx.

\section{Rated thist}

This volume is a good overview of the many uses of optical imaging to explore brain function. A wide range of methodologies discussed, and the techniques address research questions at different "levels of organization" (from intracellular imaging to systems level functional imaging of the brain). It is therefore likely that individual scientists will find only a few chapters directly relevant to their direct interests. For example, those looking at the cellular

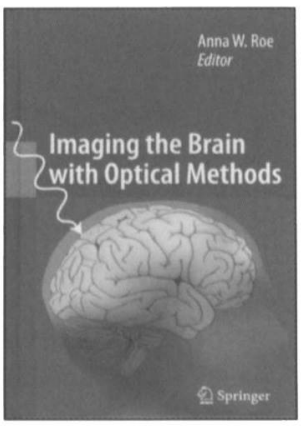
level will be interested in Chapter 2 concerning fluorescent sensors at the cell membrane level. Chapter 4 on somatosensory imaging will be of particular interest to those in exploring sensory-motor, but this section may also interest those dealing more generally with fMRI brain imaging.

Much of the book is concerned with optical imaging of intrinsic signals, with interesting applications of this technique include investigations of the representation of objects in monkey inferotemporal cortex and imaging of short-term working memory in prefrontal cortex (Chapter 5). A very interesting and useful methodological chapter is provided by Chapter 7 , relating to intrinsic signal imaging in the human cortex intraoperatively. Another clinically relevant application of optical imaging, again relying on the detection of blood flow or a BOLD (blood oxygen level dependant) like effect, is provided in Chapter 8, which nicely 